\title{
Binding Mechanism of the Model Charged Dye Carboxyfluorescein to Hyaluronan/Polylysine Multilayers
}

\author{
Vladimir Z. Prokopovic, ${ }^{\dagger, \|}$ Anna S. Vikulina, ${ }^{*},+, \|_{\odot}$ David Sustr, ${ }^{\dagger}$ Elena M. Shchukina, ${ }^{\S}$ \\ Dmitry G. Shchukin, ${ }^{\S \odot}$ and Dmitry V. Volodkin ${ }^{\ddagger}$ \\ ${ }^{\dagger}$ Branch Bioanalytics and Bioprocesses (Fraunhofer IZI-BB), Fraunhofer Institute for Cell Therapy and Immunology, Am \\ Muehlenberg 13, 14476 Potsdam-Golm, Germany \\ ${ }^{\ddagger}$ School of Science and Technology, Nottingham Trent University, Clifton Lane, NG11 8NS Nottingham, U.K. \\ ${ }^{\S}$ Stephenson Institute for Renewable Energy, University of Liverpool, L69 7ZF Liverpool, U.K.
}

\section{Supporting Information}

ABSTRACT: Biopolymer-based multilayers become more and more attractive due to the vast span of biological application they can be used for, e.g., implant coatings, cell culture supports, scaffolds. Multilayers have demonstrated superior capability to store enormous amounts of small charged molecules, such as drugs, and release them in a controlled manner; however, the binding mechanism for drug loading into the multilayers is still poorly understood. Here we focus on this mechanism using model hyaluronan/polylysine (HA/PLL) multilayers and a model charged

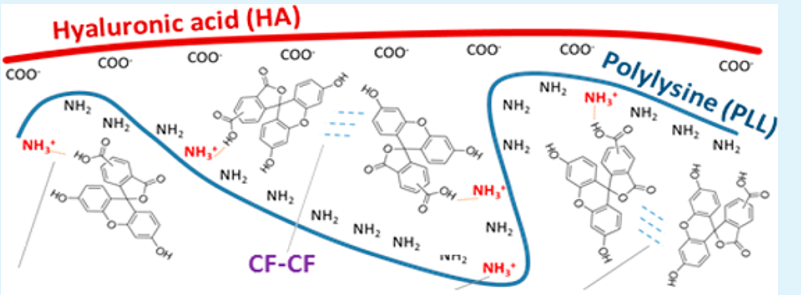

CF-PLL interaction

Cooperation

Polyadsorption dye, carboxyfluorescein (CF). We found that CF reaches a concentration of $13 \mathrm{mM}$ in the multilayers that by far exceeds its solubility in water. The high loading is not related to the aggregation of CF in the multilayers. In the multilayers, CF molecules bind to free amino groups of PLL; however, intermolecular $\mathrm{CF}-\mathrm{CF}$ interactions also play a role and (i) endow the binding with a cooperative nature and (ii) result in polyadsorption of $\mathrm{CF}$ molecules, as proven by fitting of the adsorption isotherm using the BET model. Analysis of CF mobility in the multilayers by fluorescence recovery after photobleaching has revealed that CF diffusion in the multilayers is likely a result of both jumping of CF molecules from one amino group to another and movement, together with a PLL chain being bound to it. We believe that this study may help in the design of tailor-made multilayers that act as advanced drug delivery platforms for a variety of bioapplications where high loading and controlled release are strongly desired.

KEYWORDS: layer-by-layer, adsorption isotherm, polyadsorption, diffusion, fluorescence recovery after photobleaching, hyaluronic acid

\section{INTRODUCTION}

The layer-by-layer deposition of polyelectrolyte multilayers has become very popular in the last decades due to its simplicity, low cost, automation, and reproducibility. ${ }^{1-7}$ The method is based on sequential deposition of oppositely charged polymers onto a solid surface. ${ }^{1,3-7}$ Physical-chemical properties of the multilayers can easily be adjusted to meet various requirements by proper choice of preparation conditions, variation of polyelectrolyte chain length and nature, and postmodification. ${ }^{8-11}$ Furthermore, the multilayers have attracted significant attention, since they have been shown to serve as good candidates for a number of bioapplications. ${ }^{2,12-24}$ The planar multilayers (films) and curved structures (capsules) mostly play the role of hosting reservoirs and releasing numerous biologically active molecules, such as nucleic acids, proteins, and peptides. ${ }^{2,25-30}$ Recently, a number of reports appeared to demonstrate the high capacity of multilayers to host small molecules, including drugs and dyes. ${ }^{31-33}$ This makes the multilayers very attractive as biocoatings (e.g., implant coatings) that host bioactive drugs (antibiotics, anticancer drugs, small biofactors such as growth factors, cytokines, etc.) and release them in a controlled manner, for instance, through erosion or biodegradation.

One of the most commonly used methods for loading biomolecules of interest into the multilayers is the incubation of the as-prepared multilayers in a solution of the bioactive agent. Simple dyes are often used as model molecules to mimic the behavior of a real drug. Additionally, model dyes such as carboxyfluorescein (CF) or rhodamine can be directly monitored by fluorescence or absorbance. In some cases, the capacity of a multilayer to store a dye is determined by the nature of the last polyelectrolyte layer deposited. ${ }^{34}$ Fluorescently labeled paclitaxel was homogeneously distributed throughout multilayers composed from hyaluronic acid (HA) and poly-L-lysine (PLL). ${ }^{33}$ Likewise, it was possible to load the anti-inflammatory drug diclofenac into cross-linked multilayers, and the amount of the stored drug can be tuned by the multilayer thickness/number of layers. ${ }^{35}$ Furthermore, the same

Received: August 21, 2017

Accepted: October 16, 2017

Published: October 16, 2017 
study showed that paclitaxel loaded into multilayers was effective in killing cells with an efficiency of about $90 \%$. Variation of the number of layers affects both the loading and release rate of the loaded molecules. ${ }^{36}$

High water content in the biopolymer-based multilayers, along with their structural properties, allows one to load large macromolecules such as proteins (albumin, lysozyme, others $)^{28,37}$ and nucleic acids. ${ }^{29,30}$ Full exploitation of these films for bioapplications (cell culture and tissue engineering) requires that these systems should offer a controlled release of the stored molecules. The trigger for the release can be a change in the physical-chemical properties of the multilayers or environment changes: temperature, $\mathrm{pH}$, composition, ionic strength, hydrolysis, and light. ${ }^{38-43}$ These stimuli can be employed for both short-term ${ }^{31}$ and long-term ${ }^{44}$ release. Though the possibilities for applications and control over loading and release performance are countless, the mechanism of storage/release of small molecules/drugs in the multilayers is still not well understood and a number of reports on loading and release of small drugs show high interest for this topic. $^{32-34,36,45,46}$

It has recently been demonstrated that small charged dyes can be loaded into the biopolymer-based multilayers in enormous concentrations. ${ }^{47}$ This concerns not only model dyes such as CF and rhodamine but also biologically relevant small charged molecules like adenosine triphosphate (ATP), which is of particular interest due to its well-known role in bioenergy storage and conversion. A number of reports focus on investigation of ATP loading and release into/from surface films employing the energy of ATP hydrolysis, ${ }^{48}$ electrochemically stimulated release, ${ }^{49}$ ATP-induced self-assembly, ${ }^{50}$ and loading into layered double hydroxides through intercalation of ATP and other nucleotides. ${ }^{51}$ More complex self-assembled ATP-containing structures based on metal nanoparticles and block copolymers with bioinspired binding units have also been reported. $^{52,53}$

The multilayers that have been given enormous attention as a model system are assembled from model biopolymers HA and PLL. These films can easily reach up to a few micrometers in thickness or even tens of micrometers due to their exponentiallike growth character, ${ }^{54-57}$ and thus they offer considerable reservoir capacity. ${ }^{47} \mathrm{HA} / \mathrm{PLL}$ multilayers can be used as supports for cell culture. ${ }^{58,59}$ Bearing in mind their high storage capability, these multilayers are perfect candidates as drug delivery systems for cellular applications. However, in order to comprehend their delivery potential, it is of immense importance to understand the mechanism by which a drug is withheld in the multilayers.

In this paper we focus on the binding mechanism of the small, charged, fluorescent dye CF to the HA/PLL multilayers assembled by the dipping technique. Spectral characteristics of CF along with its mobility and adsorption equilibrium capacity in the multilayers are analyzed and used as major indicators for assessment of CF binding to multilayers. We firmly believe that a better understanding of the binding mechanism of small dyes such as CF could be used to design novel, effective, polymerbased systems with tuned loading and release characteristics required for cutting-edge drug delivery and tissue-engineering applications.

\section{MATERIALS AND METHODS}

Chemicals. Sodium hyaluronate (HA; $360 \mathrm{kDa}$, \#HA500 K) was purchased from Lifecore Biomedical and Hellmanex II (\#9-307-010-
507) from Hellma GmbH. Poly-L-lysine hydrobromide (PLL; 15-30 $\mathrm{kDa}$, \#P7890), polyethylenimine (PEI; $110 \mathrm{kDa}$, \#306185), 5(6)carboxyfluorescein (CF; \#21877), TRIS, $\mathrm{NaCl}$, and $\mathrm{HCl}$ were supplied by Sigma-Aldrich. All chemicals were used as received, without further purification.

Assembly of HA/PLL Multilayers. HA/PLL films were assembled on $12 \mathrm{~mm}$ round glass slides using an automated dipcoating machine according to the protocol described elsewhere. ${ }^{59}$ Briefly, after washing with Hellmanex II, $14 \mathrm{~mm}$ round glass slides were immersed for $10 \mathrm{~min}$ in $1 \mathrm{mg} / \mathrm{mL}$ PEI, followed by washing in 10 $\mathrm{mM}$ TRIS buffer containing $15 \mathrm{mM} \mathrm{NaCl}, \mathrm{pH} 7.4$ (referred to as TRIS-buffer) three times. After that slides were immersed for $10 \mathrm{~min}$ in $0.5 \mathrm{mg} / \mathrm{mL}$ HA, washed three times in the buffer, immersed in 0.5 $\mathrm{mg} / \mathrm{mL} \mathrm{PLL}$, and again washed three times in the buffer. The HA/ PLL deposition step was repeated 24 times. The films were always terminated with PLL as the outermost layer.

Loading the Multilayers with CF and Analysis of CF Fluorescence. Loading of HA/PLL multilayers was achieved by incubating the HA/PLL in $1 \mathrm{~mL}$ of a $1 \mu \mathrm{M}$ solution of CF in TRISbuffer in darkness for $24 \mathrm{~h}$. The loading efficiency and released amount of the loaded compound were determined by analysis of the fluorescence of the supernatant.

Fluorescence emission spectra of CF solutions in pure buffer and in the buffer containing PLL were taken at $492 \mathrm{~nm}$ excitation. The concentration of CF was kept fixed at either 5 or $300 \mu \mathrm{M}$, and the PLL concentration was varied to obtain different ratios between the number of lysine units $\left(M_{\mathrm{r}}=209\right)$ in PLL and the CF molecules (molar charge PLL:CF ratio). Furthermore, fluorescence emission spectra of CF loaded in the (HA/PLL) 24 multilayers were determined; the multilayers were loaded with CF by incubation in $1 \mu \mathrm{M} \mathrm{CF}$. The measurement was done at 1 and $60 \mathrm{~min}$ after immersion of the multilayers into the TRIS-buffer in the cuvette; the moment of immersion is considered as the start of the release.

Real-time observation of the loading was done by online measurement of $\mathrm{CF}$ fluorescence in solution where the (HA/PLL) 24 multilayers were incubated. The experiment was done in quadruplicate. The amount of CF loaded in the multilayers was determined as the difference in the amount of CF measured in aliquots taken from the solution with the multilayers and the control one (without the multilayers; bar coverslip used instead).

Analysis of CF Diffusion in Multilayers by Fluorescence Recovery after Photobleaching. The diffusion coefficient and the amount of immobile fraction were assessed by evaluation of data resulting from the fluorescence recovery after photobleaching (FRAP) experiment. In general, the photobleaching may have an effect on the chemical structure of a fluorescent dye that may affect its interaction with its surroundings. However, FRAP is well-accepted method to assess the mobility of fluorescent probes like $\mathrm{CF}$, and this is why we used this method in this study. The experimental procedure and the data evaluation procedure were similar to procedures described elsewhere ${ }^{60}$ but using planar multilayers. Glass slides with deposited multilayers containing CF were mounted into a holder, covered by a layer of TRIS-buffer to protect them from drying, and placed under a confocal laser scanning microscope (Zeiss LSM 510 meta/Axiovert $200 \mathrm{M})$. Using a $63 \times / 1.4$ objective the sample was bleached by the 488 $\mathrm{nm}$ laser line of an Ar ion laser at its full power and scanned by highly attenuated laser power. The scanning area spanned $73.12 \times 73.12 \mu \mathrm{m}$ $(512 \times 512$ pixels $)$ and the bleached area spanned $73.12 \times 2.14 \mu \mathrm{m}$ $(512 \times 15$ pixels $)$. The proper $z$-position was determined as the position of the highest intensity of the fluorescence signal. The sample was primarily scanned twice, afterward bleached (by scanning the laser 50 times over the bleached area, resulting in a dip of the fluorescence signal), and then again scanned 30 times. The time span between following images was $2 \mathrm{~s}$, and the scanning time per pixel was $3.2 \mu \mathrm{s}$. The evaluation procedure of the acquired data was based on an analytical solution of Fickian diffusion. ${ }^{61}$ The evaluation of the immobile fraction amount is based on analysis of the dip depth as a function of time. The measurements were repeated six times. The details of the evaluation procedure are given below. 
The raw images from the FRAP experiment were primarily cropped (to limit edge effects) and averaged (Image J, NIH) in the direction perpendicular to the direction of diffusion. The resulting onedimensional FRAP profiles were transferred into a spreadsheet template (MS Excel) for data corrections and analysis. The set of profiles resulting from a single FRAP experiment were normalized to the prebleach fluorescence intensity, corrected to the inhomogeneity of the local fluorescence, and corrected for unwanted bleaching and edge effects in the other dimension.

Every FRAP profile is fitted by a Gaussian curve defined as

$$
I(x, t)=I_{0}(t)-A(t) \exp \left(-\frac{x^{2}}{2 w^{2}}\right)
$$

where $I(x, t)$ is the fluorescence intensity at a distance $x$ from the dip and a time point after bleaching $t, I_{0}(t)$ is the fluorescence intensity of the background (ideally its value is constant and close to 1 ), $A(t)$ is the depth of the dip at the time point $t$, and $w$ describes the width of the Gaussian between inflection points. All four parameters are free for fitting with every separate FRAP profile. The fitting is performed as a minimization of the sum of squared residuals. After fitting the Gaussian function into FRAP profiles, the diffusion coefficient $D$ is evaluated as half of the slope of the plot of $w^{2}$ versus $t$ :

$$
D=\frac{w^{2}}{2 t}
$$

Only the first three points of this plot were used for slope evaluation, because the plot tends to deviate when more than one fraction (e.g., immobile fraction) is present. No change over time of the $w^{2}$ (not equal to zero) would indicate the presence of the immobile fraction because of no recovery of the stable fluorescent profile. The slope is influenced already from the onset of the recovery process; thus, the resulting $D$ rather represents an average diffusion coefficient.

The dimensionality of diffusion $d$ may be evaluated as twice the slope of the plot $\log (A)$ versus $\log \left(t+t_{0}\right)$

$$
\log (A)=-\frac{d}{2} \log \left(t+t_{0}\right)+\text { const }
$$

where the newly appeared parameter $t_{0}$ refers to a time shift correcting deviations from the approximation of instantaneous bleaching of an infinitely thin region. The time shift can be found by varying its value until the plot $\log (A)$ versus $\log \left(t+t_{0}\right)$ becomes linear (here implemented as a maximization of the coefficient of determination of an expected linear function). Because also this plot tends to deviate when more than one fraction is present, only the first 10 points of the plot were used for evaluation of $d$.

The relative amount of the immobile fraction $K_{\text {rel }}$ is evaluated from the time development of the dip depth $A(t)$ by fitting the function

$$
A(t)=\frac{M}{4 \pi D\left(t+t_{0}\right)^{d / 2}}+K
$$

where $A(t)$ is the depth of the dip at time point $t, M$ is the overall reduction of the fluorescence intensity caused by bleaching, $D$ is the diffusion coefficient, $t$ is time after bleaching, $t_{0}$ is the time shift correction, $d$ is the dimensionality of diffusion, and $K$ is the partial depth of the dip attributed to the immobile fraction. $D$ and $d$ were taken from the previous evaluation and fixed during fitting. These values tend to be underestimated due to the influence of the immobile fraction, but their presence is essential during fitting for a good estimation of the amount of immobile fraction. The $d$ value used here is bottom-limited to a value of 1 , because values lower than 1 do not reflect reality. $M, t_{0}$, and $K$ are free for fitting. The input values $A(t)$ are acquired directly from the FRAP profiles, not from fitting the Gaussian function, because the evaluation of the immobile fraction is intended to be independent from the evaluation of $D$ as much as possible.

Afterward, $K_{\text {rel }}$ is obtained by relating $K$ to the depth of the dip just after bleaching

$$
K_{\mathrm{rel}}=\frac{K}{A_{0}(t)}
$$

where $K_{\text {rel }}$ stands for the relative amount of the immobile fraction, $K$ is the depth of the dip attributed to the immobile fraction resulting from eq 4 , and $A_{0}(t)$ is the depth of the dip just after bleaching $(t=0)$.

Such an approach to the evaluation of the immobile fraction amount expects that only two fractions are present in the sample: a single mobile fraction with certain $D$ and an immobile one. In other cases, the evaluation of the immobile fraction amount gives approximate results. When the amount of the immobile fraction is known, a contribution of this fraction can be subtracted from raw FRAP profiles. The evaluation procedure can then be repeated with the FRAP profiles free of immobile fraction. Results of this repeated evaluation describe the pure mobile fraction rather than a mixture of the mobile and the immobile fractions.

\section{RESULTS AND DISCUSSION}

Spectral Characteristics of CF in Solution and in the HA/PLL Multilayers. Our previous study has demonstrated that CF can be loaded into (HA/PLL) 24 multilayers in TRISbuffer giving high concentrations in the multilayers of at least a few millimolar. ${ }^{47}$ Incubation of excess CF with the multilayers (at concentrations up to $100 \mu \mathrm{M}$ ) under the same conditions has revealed that the saturation concentration of CF in the multilayers is $13 \mathrm{mM}$. This is far more than the CF solubility in the same buffer (about $0.5 \mathrm{mM}$ ).

In this work, we focus on the mechanism of binding of CF to multilayers in order to explain the strong accumulation of $\mathrm{CF}$ in the multilayers. The molecular structures of CF and PLL and HA biopolymers used to build the multilayers are presented in Figure S1 of the Supporting Information (SI). The dye CF possesses a carboxylic group that is deprotonated at the physiological $\mathrm{pH}$ and potentially allows the dye to interact with charged polymers through electrostatic attractive forces. Thus, throughout all experiments in this study, TRIS-buffer containing a rather low concentration of added salt $(15 \mathrm{mM})$ and possessing the physiologically relevant $\mathrm{pH} 7.4$ has been used. A low salt concentration is needed to ensure that electrostatic interactions between $\mathrm{CF}$ and permanent charges on the polymer backbone are not screened by salt counterions.

In order to investigate the interaction of CF with multilayers, fluorescent spectra of CF have been taken in the presence of the polymers from which the multilayers are made, PLL and HA. Figure S2A (SI) demonstrates that HA has no effect on the maximum of CF fluorescence and that the presence of PLL results in a shift of the maximum of fluorescence from $512 \mathrm{~nm}$ (without PLL) to $514.5 \mathrm{~nm}(0.5 \mathrm{mg} / \mathrm{mL}$ PLL solution). This can be explained by electrostatic binding of the oppositely charged CF to amino groups of PLL and no electrostatic interaction between $\mathrm{CF}$ and $\mathrm{HA}$, which both carry a negative charge. A variation of the PLL:CF molar charge ratio has shown that the red-shift of the fluorescence maximum (from 512 to $515.5 \mathrm{~nm}$ ) takes place at a PLL:CF molar charge ratio of more than 5 (Figure S2B, SI). The conditions of a high excess of PLL can simulate those in the multilayers, where PLL may be in much higher excess compared to $\mathrm{CF}$ because of the high concentration of PLL in the multilayers, which can be estimated as $250 \mathrm{mM}$ taking into account that the multilayers have about $80 \%$ of water, that the molar charge ratio between PLL and HA is about 2 , and that $46 \%$ of amino groups of PLL is free, i.e., unpaired with $\mathrm{HA}^{62}$

Apart from the binding of CF to amino groups of the PLL, $\mathrm{CF}$ is prone to aggregation, due to its core hydrophobic part of 

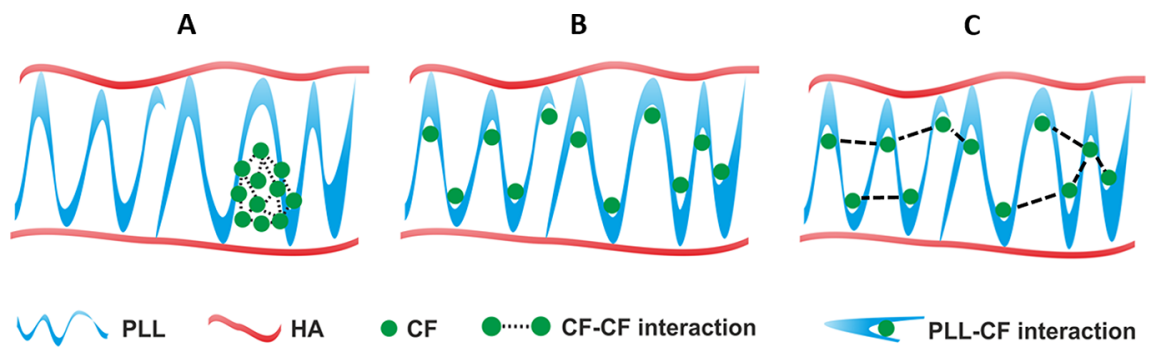

Figure 1. Schematic illustration of the three models $(A-C)$ representing binding of CF to the HA/PLL multilayers. (A) CF forms aggregates within multilayers, (B) CF is solely bound to amino groups of PLL and is equally distributed throughout the multilayers, and (C) CF is primarily bound to PLL but CF-CF interactions takes place.

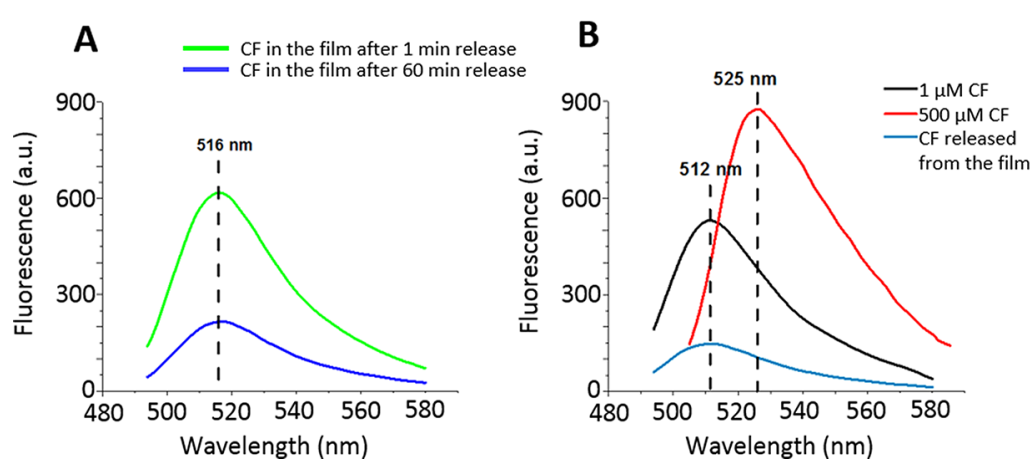

\section{C}

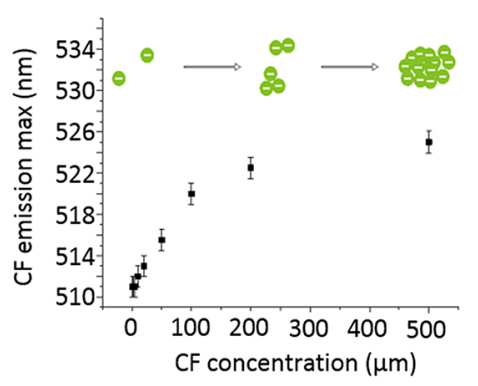

Figure 2. (A) Fluorescence spectra of CF-laden (HA/PLL) 24 multilayers incubated in TRIS-buffer for 1 and 60 min. (B) Fluorescence spectra of 1 and $500 \mu \mathrm{M}$ CF solution in TRIS-buffer as well as CF released from multilayers after 60 min incubation in the buffer. (C) Fluorescence emission maximumum as a function of CF concentration in TRIS-buffer.

the molecule. Considering this, one can propose three potential models of CF binding to multilayers, as shown in Figure 1. The model A suggests that only $\mathrm{CF}-\mathrm{CF}$ interactions take place in the multilayers. This may be reasonable because the $\mathrm{CF}$ concentration in the multilayers by far surpasses the solubility of CF in buffer, as discussed above. Such a high concentration may be explained by formation of CF aggregates if one assumes that the $\mathrm{CF}-\mathrm{CF}$ interaction is stronger than the CF-PLL interaction. Contrary to the first model, model B assumes that the energy gain for formation of CF-PLL is higher than that of the $\mathrm{CF}-\mathrm{CF}$ interaction and suggests that no aggregate formation occurs and that CF is solely bound to PLL and is also homogeneously distributed throughout the film. The third model $\mathrm{C}$ is basically a combination of the previous two models $\mathrm{A}$ and $\mathrm{B}$ and is based on the assumption that both $\mathrm{CF}-\mathrm{CF}$ and CF-PLL interactions take place.

Further, the CF-loaded multilayers and CF solutions have been analyzed to reveal which model can better describe the mechanism of binding of CF to the multilayers. Fluorescence spectra of CF in the buffer, CF loaded in the multilayers, and $\mathrm{CF}$ released from the multilayers are shown in Figure 2. One can compare the emission maximum of the spectra and take it as a measure of CF aggregation and/or CF-PLL interaction. About $70 \%$ of CF stored in the multilayers is released after $1 \mathrm{~h}$ incubation in a fresh buffer solution. ${ }^{47}$ Therefore, we have analyzed the CF spectra in the multilayers at the beginning of the release process ( $1 \mathrm{~min}$ incubation in the buffer), when almost all CF molecules should still be present in the multilayers, and after $1 \mathrm{~h}$ of incubation, when the majority of CF molecules have already left the multilayers (Figure 2A). One can see that CF molecules stored in the film have an identical emission maximum, regardless of the incubation time point, and it is about $516 \mathrm{~nm}$. In comparison, CF released to the buffer has the maximum emission of $512 \mathrm{~nm}$. This wavelength is similar to that of free CF in solution at a rather low CF concentration of $1 \mu \mathrm{M}$ and is the same as in the stock solution used in this experiment for CF loading into the multilayers (Figure 2B). However, a CF solution at the higher concentration of $500 \mu \mathrm{M}$ gives an emission maximum of 525 $\mathrm{nm}$, indicating an effect of the CF concentration on the fluorescence emission maximum.

These findings allow one to conclude that CF released from the multilayers is in a free state (nonaggregated); however, in the multilayers its emission maximum is shifted to higher wavelengths of about $516 \mathrm{~nm}$. To understand the reason for the shift, we have considered the effect of CF concentration on its emission maximum more in detail. Figure $2 \mathrm{C}$ shows that an increase of CF concentration in solution results in a progressing red-shift of emission maximum up to $525 \mathrm{~nm}$. This is obviously because of $\mathrm{CF}-\mathrm{CF}$ interactions, i.e., formation of $\mathrm{CF}$ aggregates in solution. ${ }^{63,64} \mathrm{CF}$ is becoming insoluble in TRIS-buffer at concentration of more than $500 \mu \mathrm{M}$.

The results discussed above and presented in Figure 2 clearly show that although the concentration of CF in the film is in the range of a few millimolar, it has an emission maximum at 516 $\mathrm{nm}$, much below $525 \mathrm{~nm}$, which is the emission maximum of less concentrated $0.5 \mathrm{mM}$ CF saturated solution in buffer. Additionally, there are no other peaks identified for CF stored in the film, apart from that at $516 \mathrm{~nm}$, meaning that only one CF population is present. It would then not be misleading to conclude that there are no pure $\mathrm{CF}$ aggregates in the multilayers. This means that the first model A (Figure 1) is not feasible and that it is either model $\mathrm{B}$ or $\mathrm{C}$.

In order to assess the strength of CF-PLL and CF-CF interactions and make it reflective of the $\mathrm{CF}$ microenvironment in the multilayers, we have performed experiments in solution 
where highly concentrated CF solution ( $0.3 \mathrm{mM}$, a concentration comparable to that in the multilayers) has been titrated with PLL (Figure 3). With no PLL present (at zero

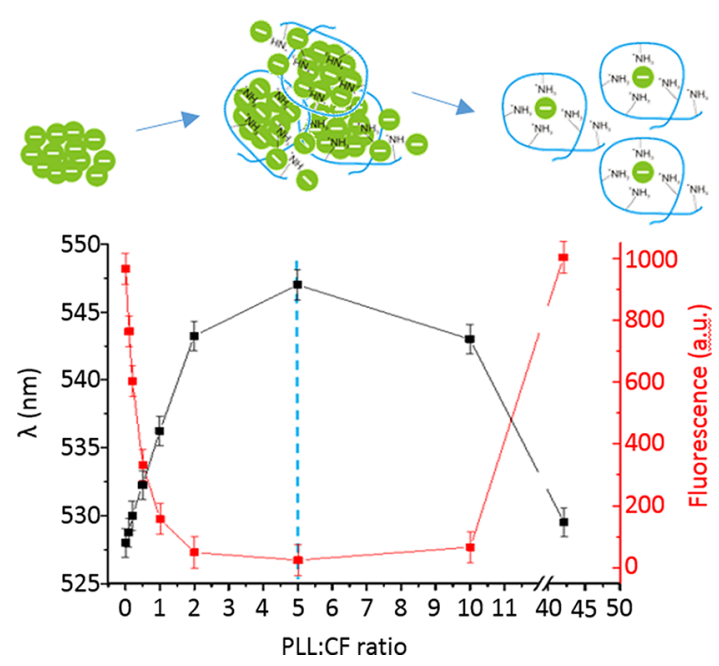

Figure 3. Dependence of the emission maximum and fluorescence intensity of a mixture of PLL and CF at different PLL:CF molar charge ratios. The interrupted vertical blue line represents the threshold at which the spectral features are reversed. Schematics above the graph demonstrate the proposed mechanism of binding of CF and PLL at increased PLL:CF molar charge ratios.

PLL:CF molar charge ratio), the maximum emission of CF is about $525 \mathrm{~nm}$, caused by CF aggregation as discussed above (Figure 2C). The wavelength maximum of emission progressively increases to about $547 \mathrm{~nm}$ until the molar charge ratio of 5. At a higher molar ratio, the emission maximum decreases back to about $530 \mathrm{~nm}$ (at the molar charge ratio of 42). The measured fluorescence intensity behaves oppositely, having a minimum at the PLL:CF molar ratio of 5 .
The results obtained are explained schematically in the figure above the graph in Figure 3. Addition of positively charged PLL induces an interaction between the negatively charged CF aggregates, making the aggregates larger. This increases the number of $\mathrm{CF}-\mathrm{CF}$ interactions, which results in a significant reduction of fluorescence due to self-quenching of $\mathrm{CF}$ molecules and a significant increase of the red-shift of the fluorescence emission maximum. Both the red-shift and selfquenching are phenomena related to intermolecular $\mathrm{CF}-\mathrm{CF}$ interactions, i.e., between $\pi$-electrons of CF molecules. ${ }^{63,64}$ At the PLL:CF molar charge ratio of more than 5, the high excess of PLL results in destruction of the formed aggregates and formation of the PLL-CF complex. This, in turn, enhances the fluorescence and reduces the red-shift due to disruption of $\mathrm{CF}$ aggregates. A PLL:CF molar charge ratio higher than 42 has not been tested because of the need for a highly concentrated PLL solution, but we hypothesize that at a higher PLL concentration the red-shift will be further reduced down to the emission maximum of $516 \mathrm{~nm}$, as has been found for PLL-CF complex at low CF concentration but high PLL excess (Figure S2B, SI).

The results above allow one to conclude that PLL-CF interaction should dominate with an excess of charged amino groups of PLL compared to carboxylic ones for CF. This is what most likely happens in the multilayers loaded with $\mathrm{CF}$, because in the multilayers the concentration of overall charges of PLL (about $250 \mathrm{mM}$ ) significantly exceeds the concentration of CF (13 mM). According to the results presented above, it can be concluded that the CF binding in the multilayers can be described by models $\mathrm{B}$ or $\mathrm{C}$ (Figure 1 ). At the same time, the CF environment in the PLL solution and inside the multilayers may be different (e.g., due to the presence of HA), and further analysis of $\mathrm{CF}-$ multilayer binding has been performed to better understand the binding mechanism and reveal the model of the binding (Figure 1).

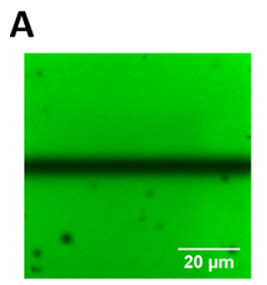

D

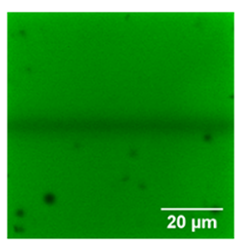

B

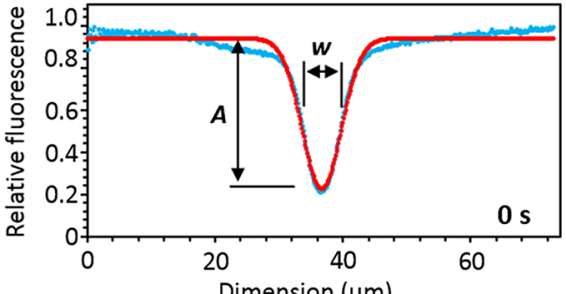

E

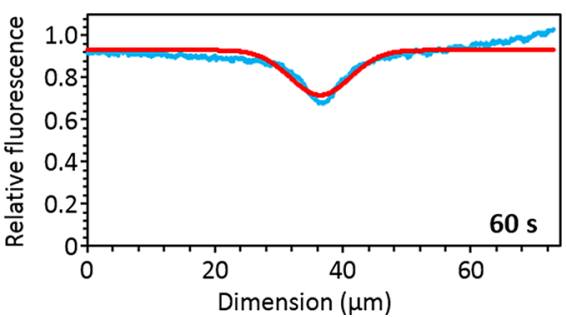

C

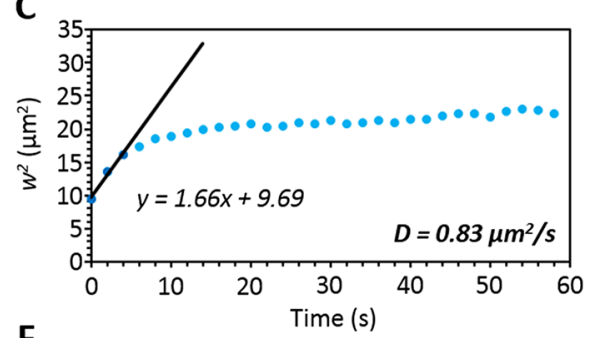

$\mathrm{F}$

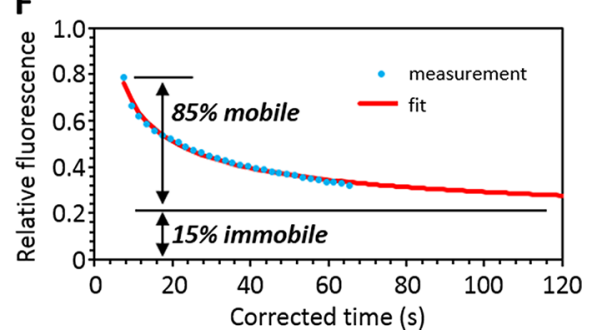

Figure 4. (A and D) Fluorescence images of CF-loaded multilayers at 0 and $60 \mathrm{~s}$ after photobleaching, respectively. (B and E) Fluorescence recovery profiles corresponding to the images A and D, respectively (perpendicular to the bleached back area in the images). The profiles are presented in blue and the fitted Gaussian functions in red. (C) Graph showing the evaluation of diffusion coefficient $(D)$. $D$ is determined as half of the slope of the first three points of the plot $w^{2}$ versus $t$ (squared width of dip versus time). At later times, the curve starts to deviate from the initial trend because of the presence of an immobile fraction. (F) A graph presenting evaluation of the amount of immobile fraction. The relative dip depth is plotted against time. Afterward a function describing this relationship (red) is fitted into the experimental data (blue) and extrapolated to infinity to get the amount of immobile fraction. Of note, small black spots in the images A and D are artifacts and are not related to the FRAP procedure. 
Table 1. Comparison of Six Adsorption Models ${ }^{66}$ Used To Fit Adsorption Isotherms for CF Loading into (HA/PLL) 24 Multilayers (Figure 5) ${ }^{a}$

\begin{tabular}{|c|c|c|c|}
\hline model & mathematical eq & fitted parameters & $R^{2}$ \\
\hline Langmuir & $C_{\text {film }}=\frac{C_{\text {film max }} K C_{\text {sol }}}{1+K C_{\text {sol }}}$ & $K=0.35 \pm 0.13$ & 0.538 \\
\hline Freundlich & $C_{\text {film }}=K C_{\text {sol }}^{1 / n}$ & $\begin{array}{l}K=224 \pm 210 \\
n=0.5 \pm 0.1\end{array}$ & 0.899 \\
\hline Langmuir-Freundlich & $C_{\text {film }}=\frac{C_{\text {film max }}\left(K C_{\text {sol }}\right)^{1 / n}}{1+\left(K C_{\text {sol }}\right)^{1 / n}}$ & $\begin{array}{l}K=(1.4 \pm 1.8) \times 10^{-4} \\
n=0.19 \pm 0.12\end{array}$ & 0.854 \\
\hline Temkin & $C_{\text {film }}=A \ln C_{\mathrm{sol}}+B$ & $\begin{array}{l}A=3924 \pm 895 \\
B=724 \pm 258\end{array}$ & 0.767 \\
\hline sigmoidal Langmuir & $C_{\text {film }}=\frac{C_{\text {film max }} K C_{\text {sol }}}{1+K C_{\text {sol }}+S / C_{\text {sol }}}$ & $K=(3.8 \pm 0.2) \times 10^{4}$ & 0.737 \\
\hline BET & $C_{\text {film }}=\frac{C_{\text {mono }} K C_{\text {sol }} C_{\text {sat }}}{\left(C_{\mathrm{sat}}+(K-1) C_{\mathrm{sol}}\right)\left(C_{\mathrm{sat}}-C_{\mathrm{sol}}\right)}$ & $\begin{array}{l}S=(3.6 \pm 2.8) \times 10^{5} \\
C_{\text {mono }}=1909 \pm 787 \mu \mathrm{M}\end{array}$ & 0.966 \\
\hline & & $\begin{array}{l}K=27 \pm 8 \\
C_{\mathrm{sat}}=7.5 \pm 0.4 \mu \mathrm{M}\end{array}$ & \\
\hline
\end{tabular}

${ }^{a} C_{\text {sol }}$ is the concentration of $\mathrm{CF}$ in solution; $C_{\text {film }}$ is the concentration of CF in the film; $C_{\text {film max }}$ is the maximum concentration of CF in the film (this parameter was fixed for the fitting procedure at $13.1 \pm 1.2 \mathrm{mM}$ based on the experimental results); $C_{\text {mono }}$ is the maximum concentration of CF per one deposited layer; $C_{\text {sat }}$ is the maximum concentration of $C F$ in solution; and $K, A, B, S$, and $n$ are parameters of the fitting. Fitted parameters are presented as the average $\pm \mathrm{SD}$ for $n=4$ experiments.
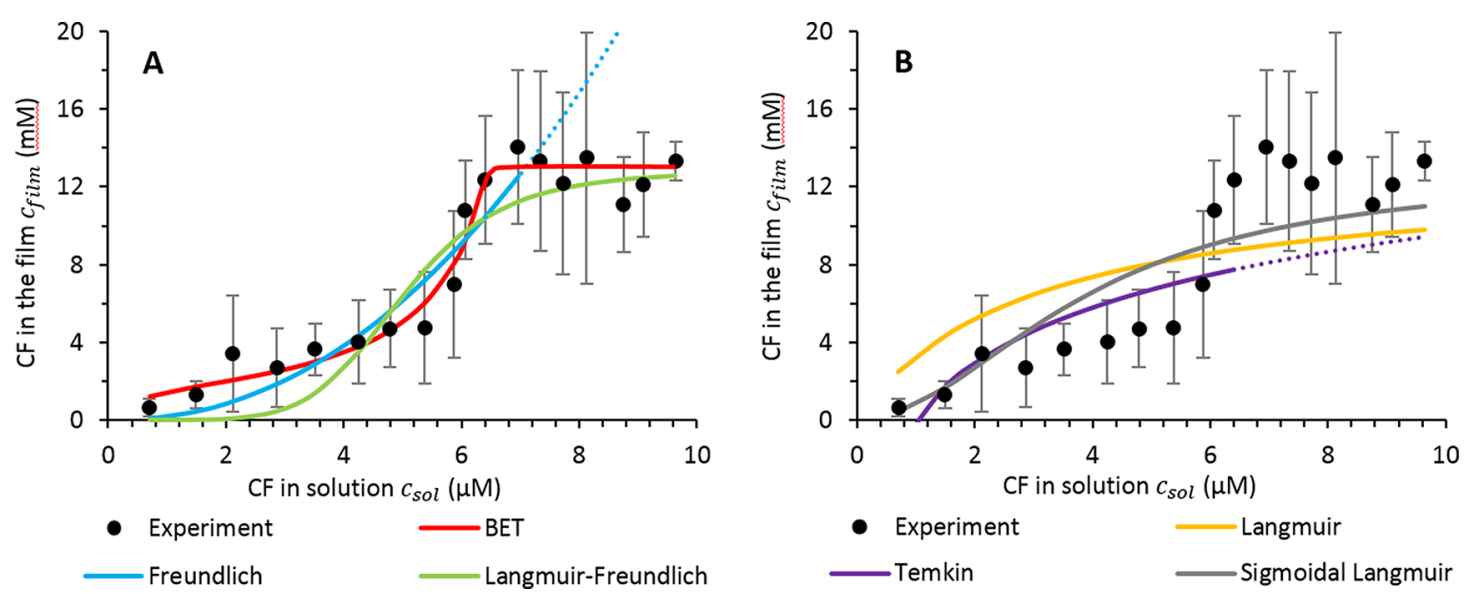

Figure 5. Fitting of the adsorption isotherm (SD are given for $n=4)$ obtained for CF loading into (HA/PLL) 24 multilayers by (A) BET, Freundlich, and Langmuir-Freundlich models and (B) Langmuir, sigmoidal Langmuir, and Temkin models.

Analysis of CF Diffusion into the Mutilayers. A series of FRAP experiments have been conducted to check the mobility of CF in the film. This may help to explain the interaction of $\mathrm{CF}$ and PLL. The mean CF diffusion coefficient $\left(D_{\mathrm{CF}}\right)$ and the amount of immobile CF fraction were estimated from these experiments (Figure 4). The resulting diffusion coefficient is an average diffusion coefficient of all CF fractions presented in our sample and was found to be $1.3 \pm 0.6 \mu \mathrm{m}^{2} / \mathrm{s}$. The amount of immobile fraction refers to the CF fraction that seems to be immobile on the time scale of our experiment $\left(D<0.01 \mu \mathrm{m}^{2} /\right.$ s). The immobile fraction was found to be about $9 \pm 10 \%$ of the total CF presented in the multilayers. One can compare the found $D_{\mathrm{CF}}$ in multilayers and $D$ of PLL analyzed by a similar FRAP approach. ${ }^{65}$ The mean $D_{\mathrm{CF}}$ is similar to the $D_{\mathrm{PLL}}$ for the fastest PLL fraction, which is about $1 \mu \mathrm{m}^{2} / \mathrm{s}$. Three PLL diffusive fractions were found in the multilayers. ${ }^{65}$ Slower fractions of PLL have also been identified, but their contribution into the mean $D_{\mathrm{PLL}}$ measured will not be significant because the second diffusive fraction is much slower (about $0.1 \mu \mathrm{m}^{2} / \mathrm{s}$ ) than the fastest one. The third fraction of PLL is immobile ( $D$ below $0.001 \mu \mathrm{m}^{2} / \mathrm{s}$ ), but its content in the multilayers $(30-40 \%)$ is much higher than that for CF found here $(9 \%)$.

The results above suggest that CF diffusion in the multilayers is most probably a result of both (i) free diffusion of $\mathrm{CF}$ through jumping of CF molecules from one PLL backbone to another and (ii) diffusion of CF molecules together with PLL chains (bound to the PLL backbone). These results corroborate well with our previous findings based on analysis of CF release from multilayers as a function of the multilayer properties. $^{47}$

To further understand the binding of CF to the multilayers, the kinetics of $\mathrm{CF}$ interaction with the multilayers has been assessed at increased CF concentrations (Figure S3, SI). From 

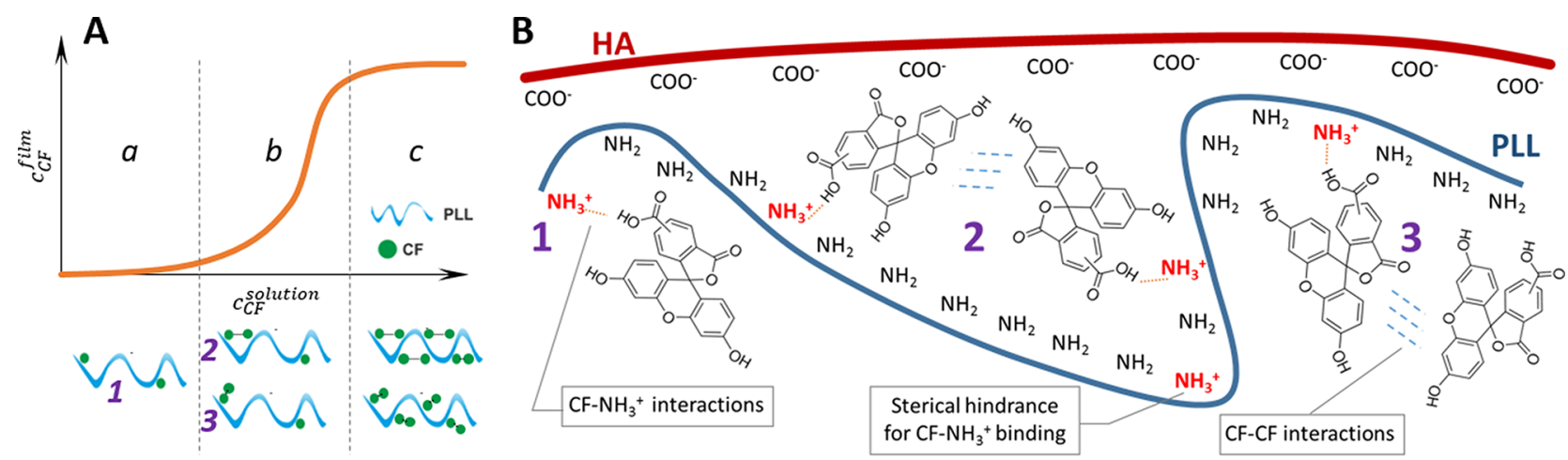

Figure 6. (A) S-shaped isotherm of CF adsorption into HA/PLL multilayers and schematics of CF-PLL and CF-CF interactions in the multilayers as a function of the CF concentration in solution. (B) Schematic presentation of the PLL backbone interacting with CF molecules. Both ion pairs and intermolecular $\mathrm{CF}-\mathrm{CF}$ interactions play a role; however, not all the amino groups can be bound to CF, due to either a sterical factor or the reduced number of charged amino groups due to the significantly lower apparent $\mathrm{p} K_{\mathrm{a}}$ of PLL in the multilayers compared to that in solution.

these results, the adsorption isotherms have been constructed and fitted by six well-known adsorption models based on equilibrium adsorption process, i.e., Langmuir, Freundlich, Langmuir-Freundlich, Temkin, sigmoidal Langmuir, and Brunauer-Emmett-Teller (BET) models ${ }^{66}$ (Table 1). The main characteristics of the models are summarized in Table $S 1$ (SI). Briefly, only the Langmuir model is based on the assumption of formation of a monolayer (monoadsorption), and all other models assume that each adsorbed molecule provides a new binding site for another molecule to be adsorbed. All other five polyadsorption models are based on the assumption that either adsorbate (CF) molecules do not interact with each other or the interaction of adsorbate (CF) and adsorbent (HA/PLL film) has a cooperative or competitive nature. It is important to note that for these models only Langmuir and BET equations (Table 1) can be derived on the basis of statistical thermodynamics, while the other models are empirical.

The data used for fitting was the average of the quadruplicate measurement. As the measure of the fit we used the coefficient of determination $\left(R^{2}\right)$ in order to reveal the model that better fits the experimental results ( $R^{2}$ closest to a unit). $R^{2}$ was calculated using the equation for the general case

$$
R^{2}=1-\frac{\mathrm{SS}_{\mathrm{res}}}{\mathrm{SS}_{\mathrm{tot}}}
$$

where $\mathrm{SS}_{\text {res }}$ is the sum of squared residuals, $\mathrm{SS}_{\text {res }}=\sum_{i}\left(y_{i}-f_{i}\right)^{2}$. It is a parameter to be minimized during the fitting procedure. $y$ stands for input data values, and $f$ stands for predicted values. $\mathrm{SS}_{\text {tot }}$ is the total sum of squares, $\mathrm{SS}_{\text {tot }}=\sum_{i}\left(y_{i}-y_{\mathrm{av}}\right)^{2} \cdot y$ stands for input data values, and $y_{\mathrm{av}}$ stands for the average value of variable $y$ in our sample. This parameter is proportional to the variance of $y$.

It should be noted that the experimental adsorption isotherm has a clearly defined region of saturation (Figure 5). This was taken into account by fixing the parameter $C_{\text {film max }}$ at $13.1 \pm 1.2$ $\mathrm{mM}$. By the same token, Freundlich, Temkin, and BET equations were fitted until the saturation plateau.

Figure 5A presents the fitting of experimental results of the CF loading into the multilayers by the three above-mentioned adsorption models that provided the best fitting results (correlation coefficient $R>0.90$ ), i.e., BET, Freundlich, and Langmuir-Freundlich models. Adsorption isotherms for the three other models (Langmuir, sigmoidal Langmuir, and Temkin), which show $R<0.90$, are given in Figure 5B.
The best fit was found for the BET model $\left(R^{2}=0.966\right)$. This indicates that the interaction of $\mathrm{CF}$ with (HA/PLL) 24 multilayers is likely according to assumptions on which the BET model is based. Considering the five general types of BET adsorption isotherms that are known (Table S2, SI), CF loading into the multilayers can be described in the best way by the S-shaped type $\mathrm{V}$ adsorption isotherm. The S-type of isotherm suggests cooperative adsorption, when the binding of adsorbate molecules to the adsorbent depends on the concentration of adsorbed molecules; in other words, binding of two adsorbate molecules to the adsorbent is not independent. ${ }^{67}$ The binding of one molecule increases the affinity for the binding of the next molecule. ${ }^{67}$ At the same time, the type $\mathrm{V}$ BET isotherm refers to the polyadsorption of $\mathrm{CF}$ molecules to equivalent binding cites. This means that each CF molecule adsorbed to a PLL backbone provides a new site for the adsorption of the molecule in the layer above it, and the anchorage to two types of binding cites $\left(\mathrm{NH}_{3}{ }^{+}\right.$group or $\mathrm{CF}$ molecule) is characterized by the same or close adsorption energies; i.e., binding sites within the film are equivalent. The proposed integrated mechanism of adsorption is schematically represented in Figure 6. This proves that the most suitable model to describe binding of CF to HA/PLL multilayers is model $\mathrm{C}$ proposed in Figure 1.

According to the schematics in Figure 6, first molecules of CF loaded into the multilayers bind to amino groups of PLL and do not interact with each other (Figure 6, case 1). This type of binding is driven through the PLL-CF interaction and is apparently predominant at a low concentration of $\mathrm{CF}$ in solution (zone a). As CF molecules keep entering the film (zone b), apart from binding to PLL, they interact with each other. CF molecules that are anchored to the film either strengthen the binding of upcoming CF molecules via a CF$\mathrm{CF}$ interaction (cooperation, case 2) or act as new binding cites for CF molecules that are coming from the solution (case 3 ). At the same time, there is no clustering of CF molecules to each other (aggregation), as proven earlier (Figure 2), the interaction is presumably between two CF molecules. At high concentrations of CF (zone c), all possible binding sites will be occupied, resulting in saturation of the multilayers with $\mathrm{CF}$ molecules and reaching a plateau on the adsorption isotherm. Figure 6 illustrates two extreme cases, where either only the cooperative mechanism (case 2 ) or only polyadsorption (case 3 ) is realized. However, we suppose that both cases take place simultaneously. 
Interestingly, the number of occupied binding sites is less than the total number of free amino groups of PLL in the multilayers. One can notice that the molar charge ratio PLL:CF is about 20 [CF maximum concentration in the multilayers is $13 \mathrm{mM}$ (Figure 5) and the total concentration of amino groups of PLL is about $250 \mathrm{mM}$, as we calculated above]. This can be explained either by sterical factors or the reduced number of charged amino groups available for interaction with $\mathrm{CF}$ in the multilayers. A sterical factor may mean that the CF-PLL interaction can only be realized if CF can find a PLL amino group properly oriented in the space to make an ion pair with it (Figure 6B). One cannot expect significant sterical limitations for CF molecule to reach a backbone of PLL because the HA/ PLL multilayers are highly hydrated (about $80 \%$ of water) and about half $(46 \%)$ of the amino groups of PLL are unpaired with $\mathrm{HA}$ (free to interact with $\mathrm{CF}$ ). ${ }^{62}$ In addition, $\mathrm{CF}$ is a small, compact molecule, allowing free diffusion in the hydrated and bulky multilayers. However, the PLL is a highly flexible polymer, and it can intertwine, making the access of CF to the amino groups of PLL difficult.

A limited number of charged amino groups can be another reason to be considered. The reduced $\mathrm{pK}$ a of PLL in HA/PLL multilayers has been reported. ${ }^{68}$ Both $\mathrm{HA}$ and PLL are becoming weaker acid and weaker base in the multilayers compared to their strength in solution. The changes in the $\mathrm{p} K_{\mathrm{a}}$ are significant and may be a few $\mathrm{pH}$ units. The authors ${ }^{68}$ have reported that in the multilayers assembled at $\mathrm{pH}$ 7.0, HA changes its $\mathrm{p} K_{\mathrm{a}}$ from 3.8 to 4.9 and the $\mathrm{p} K_{\mathrm{a}}$ of PLL is significantly decreased from 9.4 to 6.8 . This is related to the ability of the polymers to change their secondary structure and increase a degree of conformational order upon adsorption to the multilayers. At $\mathrm{pH} 7.0$ in the multilayers $\mathrm{HA}$ remains still fully negatively charged ( $\mathrm{p} K_{\mathrm{a}}$ much below 7.0$)$ but the charge density of PLL can be significantly reduced due to its much lower $\mathrm{p} K_{\mathrm{a}}$ value in multilayers, i.e., 6.8. This may explain why CF does not interact with all the available amino groups of PLL in the multilayer but only with a rather small number of the groups that still carry a charge. One can speculate that both the sterical factor and reduction of the apparent $\mathrm{p} K_{\mathrm{a}}$ of PLL can play a role.

We believe that the physical-chemical approaches employed here to understand CF interaction with HA/PLL multilayers may be helpful to assess the interaction of real drugs with multilayer films and thus open new perspectives to make advanced biocoatings with tuned loading and release of drugs. One would expect that some real drugs, such as the anticancer drug doxorubicin, may possess stronger intermolecular interaction between drug molecules due to the hydrophobic nature of the drug and lack of charge. The same approach used here may be used for doxorubicin loaded into the multilayers. The aspects of drug or biomolecule (e.g., ATP, doxorubicin) release from HA/PLL multilayers will be referred to in our upcoming research.

Understanding the drug-multilayer interaction is a key for the design of new multilayer-based drug delivery systems, because this interaction will drive drug release performance. The proper choice of the multilayer constituents (two or more polymers such as blend multilayers) will dictate the release rate, allowing one to get the desired release profile, e.g., burst or prolonged release or a more complicated profile. In addition, knowledge of the molecular binding to the multilayers may help one to engineer free-standing multilayer shells enabling protective characteristics ${ }^{69}$ through controlled shell perme- ability governed by the interaction of molecules from outside with the capsule shell. We believe that the results of this study performed at a salt concentration below the physiological salt concentration will be helpful for the design of novel multilayerbased drug delivery systems, because the salt concentration in the multilayers shall be defined by uncompensated charges in the polymer network of the multilayers if enough salt (to form counterions) is provided, as in this study. However, interesting aspects related to the salt concentration inside and outside the multilayers and the effect salt can have on drug loading/release will be considered separately in our future research.

\section{CONCLUSIONS}

In this study we focused on the binding mechanism of the small, charged dye CF to biopolymer-based multilayers, namely, $(\mathrm{HA} / \mathrm{PLL})_{24}$ multilayers. CF is a popular fluorescent dye often used as a model drug. CF can spontaneously be loaded into the multilayers with a very high partitioning coefficient of about $10^{4}$, reaching a dye concentration in the film of up to $13 \mathrm{mM}$, which is much more than the dye solubility in solution $(0.5$ $\mathrm{mM})$. The CF loading is driven by binding of CF molecules to free amino groups of PLL in the multilayers. In the multilayers $\mathrm{CF}-\mathrm{CF}$ interactions take place but do not dominate over CFPLL interactions, giving no aggregates of $\mathrm{CF}$, as found by analysis of CF fluorescence spectra in the multilayers and in solution. The binding of CF to multilayers is cooperative, as indicated by the $\mathrm{S}$-shaped adsorption isotherm. This means that binding of one CF molecule to the multilayer affects the binding of another molecule, making the binding of new coming molecules more favorable. At the same time, anchorage of CF takes place in accordance with the BET type V polyadsorption model, meaning that $\mathrm{CF}$ molecules that are stored in the HA/PLL film play a role as new binding sites for new CF molecules. Both mechanisms of adsorption are driven by $\mathrm{CF}-\mathrm{CF}$ interactions that enhance the binding affinity and strengthen the $\mathrm{CF}$-multilayer interaction. Analysis of $\mathrm{CF}$ mobility in the multilayers by FRAP has revealed that more than $90 \%$ of CF molecules diffuse very fast (diffusion coefficient $D$ of about $1 \mu \mathrm{m}^{2} / \mathrm{s}$ ) and the rest is immobile. As reported in the literature, mobile PLL has a similar $D$ in the multilayers but a much higher content of immobile fraction $(30-40 \%) .{ }^{65} \mathrm{On}$ the basis of this, one can conclude that CF diffuses in the multilayers through both (i) jumping from one amino group to another (at one or different PLL chains) and (ii) diffusion together with PLL chains being bound to the chains. This study provides new insights into the multilayer-drug interactions using the model fluorescent dye CF. We believe that the developed approach and knowledge received can be further extended and utilized for understanding the mechanism of loading and release of biologically relevant drugs. Elucidation of the storage mechanisms would open a new way that would provide one with knowledge to precisely design tailor-made multilayers well-suited for applications in drug protection, separation, and controlled release.

\section{ASSOCIATED CONTENT}

\section{Supporting Information}

The Supporting Information is available free of charge on the ACS Publications website at DOI: 10.1021/acsami.7b12449.

Fluorescence emission spectra at different PLL:CF ratios; molecular structures of CF, PLL, and HA; fluorescence 
of HA/PLL multilayers during CF loading; and details of the mathematical modeling of CF adsorption (PDF)

\section{AUTHOR INFORMATION}

\section{Corresponding Author}

*E-mail: anna.vikulina@ntu.ac.uk. Tel: +44 1158488062.

\section{ORCID}

Anna S. Vikulina: 0000-0001-9427-2055

Dmitry G. Shchukin: 0000-0002-2936-804X

Dmitry V. Volodkin: 0000-0001-7474-5329

\section{Author Contributions}

"V.Z.P. and A.S.V. contributed equally to this work. The manuscript was written through contributions of all authors. All authors have given approval to the final version of the manuscript.

\section{Notes}

The authors declare no competing financial interest.

\section{ACKNOWLEDGMENTS}

D.V.V. acknowledges the Alexander von Humboldt Foundation (Sofja Kovalevskaja Program), the DFG grant VO 1716/2-1, and Material Research Seed-Corn Fund from NTU for financial support. D.S. and E.M.S. acknowledge the ERC Consolidator2014 grant ENERCAPSULE for financial support.

\section{ABBREVIATIONS USED}

$\mathrm{HA}=$ hyaluronic acid

PLL $=$ poly-L-lysine

$\mathrm{CF}=$ carboxyfluorescein

ATP $=$ adenosine triphosphate

$\mathrm{PEI}=$ polyethylenimine

$\mathrm{FRAP}=$ fluorescence recovery after photobleaching

$\mathrm{BET}=$ Brunauer-Emmett-Teller

\section{REFERENCES}

(1) Decher, G.; Hong, J. D. Buildup of Ultrathin Multilayer Films by a Self-assembly Process. 1. Consecutive Adsorption of Anionic and Cationic Bipolar Amphiphiles on Charged Surfaces. Makromol. Chem., Macromol. Symp. 1991, 46, 321-327.

(2) Volodkin, D.; Skirtach, A.; Mohwald, H. LbL Films as Reservoirs for Bioactive Molecules. Adv. Polym. Sci. 2010, 240, 135-161.

(3) de Villiers, M. M.; Otto, D. P.; Strydom, S. J.; Lvov, Y. M. Introduction to Nanocoatings Produced by Layer-by-layer (LbL) Selfassembly. Adv. Drug Delivery Rev. 2011, 63, 701-715.

(4) Aggarwal, N.; Altgarde, N.; Svedhem, S.; Zhang, K.; Fischer, S.; Groth, T. Effect of Molecular Composition of Heparin and Cellulose Sulfate on Multilayer Formation and Cell Response. Langmuir 2013, 29, 13853-13864.

(5) Aggarwal, N.; Altgarde, N.; Svedhem, S.; Michanetzis, G.; Missirlis, Y.; Groth, T. Tuning Cell Adhesion and Growth on Biomimetic Polyelectrolyte Multilayers by Variation of $\mathrm{pH}$ During Layer-by-Layer Assembly. Macromol. Biosci. 2013, 13, 1327-1338.

(6) Butergerds, D.; Cramer, C.; Schonhoff, M. pH-Dependent Growth Laws and Viscoelastic Parameters of Poly-L-Lysine/Hyaluronic Acid Multilayers. Adv. Mater. Interfaces 2017, 4, 1600592.

(7) Lavalle, P.; Voegel, J. C.; Vautier, D.; Senger, B.; Schaaf, P.; Ball, V. Dynamic Aspects of Films Prepared by a Sequential Deposition of Species: Perspectives for Smart and Responsive Materials. Adv. Mater. 2011, 23, 1191-1221.

(8) Borges, J.; Mano, J. F. Molecular Interactions Driving the Layerby-Layer Assembly of Multilayers. Chem. Rev. 2014, 114, 8883-8942.

(9) Klitzing, R. v. Internal Structure of Polyelectrolyte Multilayer Assemblies. Phys. Chem. Chem. Phys. 2006, 8, 5012-5033.
(10) Dubas, S. T.; Schlenoff, J. B. Swelling and Smoothing of Polyelectrolyte Multilayers by Salt. Langmuir 2001, 17, 7725-7727.

(11) Volodkin, D.; von Klitzing, R.; Moehwald, H. Polyelectrolyte Multilayers: Towards Single Cell Studies. Polymers 2014, 6, 15021527.

(12) Tang, Z. Y.; Wang, Y.; Podsiadlo, P.; Kotov, N. A. Biomedical Applications of Layer-by-layer Assembly: From Biomimetics to Tissue Engineering. Adv. Mater. 2006, 18, 3203-3224.

(13) Pavlukhina, S.; Sukhishvili, S. Polymer Assemblies for Controlled Delivery of Bioactive Molecules from Surfaces. Adv. Drug Delivery Rev. 2011, 63, 822-836.

(14) Knopf-Marques, H.; Pravda, M.; Wolfova, L.; Velebny, V.; Schaaf, P.; Vrana, N. E.; Lavalle, P. Hyaluronic Acid and Its Derivatives in Coating and Delivery Systems: Applications in Tissue Engineering, Regenerative Medicine and Immunomodulation. Adv. Healthcare Mater. 2016, 5, 2841-2855.

(15) Knopf-Marques, H.; Singh, S.; Htwe, S. S.; Wolfova, L.; Buffa, R.; Bacharouche, J.; Francius, G.; Voegel, J. C.; Schaaf, P.; Ghaemmaghami, A. M.; Vrana, N. E.; Lavalle, P. Immunomodulation with Self-Crosslinked Polyelectrolyte Multilayer-Based Coatings. Biomacromolecules 2016, 17, 2189-2198.

(16) Boudou, T.; Crouzier, T.; Ren, K. F.; Blin, G.; Picart, C. Multiple Functionalities of Polyelectrolyte Multilayer Films: New Biomedical Applications. Adv. Mater. 2010, 22, 441-467.

(17) Prokopovic, V. Z.; Vikulina, A. S.; Sustr, D.; Duschl, C.; Volodkin, D. Biodegradation-Resistant Multilayers Coated with Gold Nanoparticles. Toward a Tailor-made Artificial Extracellular Matrix. ACS Appl. Mater. Interfaces 2016, 8, 24345-24349.

(18) Vogt, C.; Ball, V.; Mutterer, J.; Schaaf, P.; Voegel, J. C.; Senger, B.; Lavalle, P. Mobility of Proteins in Highly Hydrated Polyelectrolyte Multilayer Films. J. Phys. Chem. B 2012, 116, 5269-5278.

(19) Muzzio, N. E.; Pasquale, M. A.; Gregurec, D.; Diamanti, E.; Kosutic, M.; Azzaroni, O.; Moya, S. E. Polyelectrolytes Multilayers to Modulate Cell Adhesion: A Study of the Influence of Film Composition and Polyelectrolyte Interdigitation on the Adhesion of the A549 Cell Line. Macromol. Biosci. 2016, 16, 482-495.

(20) Muzzio, N. E.; Gregurec, D.; Diamanti, E.; Irigoyen, J.; Pasquale, M. A.; Azzaroni, O.; Moya, S. E. Thermal Annealing of Polyelectrolyte Multilayers: An Effective Approach for the Enhancement of Cell Adhesion. Adv. Mater. Interfaces 2017, 4, 1600126.

(21) Zhou, G. Y.; Niepel, M. S.; Saretia, S.; Groth, T. Reducing the Inflammatory Responses of Biomaterials by Surface Modification with Glycosaminoglycan Multilayers. J. Biomed. Mater. Res., Part A 2016, 104, 493-502.

(22) Silva, J. M.; Reis, R. L.; Mano, J. F. Biomimetic Extracellular Environment Based on Natural Origin Polyelectrolyte Multilayers. Small 2016, 12, 4308-4342.

(23) Costa, R. R.; Mano, J. F. Polyelectrolyte Multilayered Assemblies in Biomedical Technologies. Chem. Soc. Rev. 2014, 43, 3453-3479.

(24) Niepel, M. S.; Mano, J. F.; Groth, T. Effect of Polyelectrolyte Multilayers Assembled on Ordered Nanostructures on Adhesion of Human Fibroblasts. ACS Appl. Mater. Interfaces 2016, 8, 2514225151.

(25) Guillame-Gentil, O.; Semenov, O.; Roca, A. S.; Groth, T.; Zahn, R.; Voros, J.; Zenobi-Wong, M. Engineering the Extracellular Environment: Strategies for Building 2D and 3D Cellular Structures. Adv. Mater. 2010, 22, 5443-5462.

(26) Volodkin, D. V.; Balabushevitch, N. G.; Sukhorukov, G. B.; Larionova, N. I. Model System for Controlled Protein Release: pHSensitive Polyelectrolyte Microparticles. STP Pharma Sci. 2003, 13, $163-170$.

(27) Balabushevich, N. G.; Pechenkin, M. A.; Shibanova, E. D.; Volodkin, D. V.; Mikhalchik, E. V. Multifunctional Polyelectrolyte Microparticles for Oral Insulin Delivery. Macromol. Biosci. 2013, 13, $1379-1388$

(28) Uhlig, K.; Madaboosi, N.; Schmidt, S.; Jaeger, M. S.; Rose, J.; Duschl, C.; Volodkin, D. V. 3D Localization and Diffusion of Proteins in Polyelectrolyte Multilayers. Soft Matter 2012, 8, 11786-11789. 
(29) Jewell, C. M.; Lynn, D. M. Multilayered Polyelectrolyte Assemblies as Platforms for the Delivery of DNA and Other Nucleic Acid-Based Therapeutics. Adv. Drug Delivery Rev. 2008, 60, 979-999.

(30) Paulraj, T.; Feoktistova, N.; Velk, N.; Uhlig, K.; Duschl, C.; Volodkin, D. Microporous Polymeric 3D Scaffolds Templated by the Layer-by-Layer Self-Assembly. Macromol. Rapid Commun. 2014, 35, $1408-1413$

(31) Chuang, H. F.; Smith, R. C.; Hammond, P. T. Polyelectrolyte Multilayers for Tunable Release of Antibiotics. Biomacromolecules 2008, 9, 1660-1668.

(32) Schneider, A.; Vodouhe, C.; Richert, L.; Francius, G.; Le Guen, E.; Schaaf, P.; Voegel, J. C.; Frisch, B.; Picart, C. Multifunctional Polyelectrolyte Multilayer Films: Combining Mechanical Resistance, Biodegradability, and Bioactivity. Biomacromolecules 2007, 8, 139-145.

(33) Vodouhe, C.; Le Guen, E.; Garza, J. M.; Francius, G.; Dejugnat, C.; Ogier, J.; Schaaf, P.; Voegel, J. C.; Lavalle, P. Control of Drug Accessibility on Functional Polyelectrolyte Multilayer Films. Biomaterials 2006, 27, 4149-4156.

(34) Kharlampieva, E.; Sukhishvili, S. A. Release of a Dye from Hydrogen-Bonded and Electrostatically Assembled Polymer Films Triggered by Adsorption of a Polyelectrolyte. Langmuir 2004, 20, 9677-9685.

(35) Schneider, A.; Picart, C.; Senger, B.; Schaaf, P.; Voegel, J. C.; Frisch, B. Layer-by-Layer Films from Hyaluronan and Amine-Modified Hyaluronan. Langmuir 2007, 23, 2655-2662.

(36) Berg, M. C.; Zhai, L.; Cohen, R. E.; Rubner, M. F. Controlled Drug Release from Porous Polyelectrolyte Multilayers. Biomacromolecules 2006, 7, 357-364.

(37) Szyk, L.; Schaaf, P.; Gergely, C.; Voegel, J. C.; Tinland, B. Lateral Mobility of Proteins Adsorbed on or Embedded in Polyelectrolyte Multilayers. Langmuir 2001, 17, 6248-6253.

(38) De Geest, B. G.; Sanders, N. N.; Sukhorukov, G. B.; Demeester, J.; De Smedt, S. C. Release Mechanisms for Polyelectrolyte Capsules. Chem. Soc. Rev. 2007, 36, 636-649.

(39) Zhu, Z. C.; Gao, N.; Wang, H. J.; Sukhishvili, S. A. Temperature-Triggered On-Demand Drug Release Enabled by Hydrogen-Bonded Multilayers of Block Copolymer Micelles. J. Controlled Release 2013, 171, 73-80.

(40) Borges, J.; Rodrigues, L. C.; Reis, R. L.; Mano, J. F. Layer-byLayer Assembly of Light-Responsive Polymeric Multilayer Systems. Adv. Funct. Mater. 2014, 24, 5624-5648.

(41) Volodkin, D.; Skirtach, A.; Madaboosi, N.; Blacklock, J.; von Klitzing, R.; Lankenau, A.; Duschl, C.; Mohwald, H. IR-light Triggered Drug Delivery from Micron-Sized Polymer Biocoatings. J. Controlled Release 2010, 148, e70-e71.

(42) Volodkin, D.; Skirtach, A.; Mohwald, H. Bioapplications of Light-Sensitive Polymer Films and Capsules Assembled Using the Layer-by-Layer Technique. Polym. Int. 2012, 61, 673-679.

(43) Parakhonskiy, B. V.; Yashchenok, A. M.; Mohwald, H.; Volodkin, D.; Skirtach, A. G. Release from Polyelectrolyte Multilayer Capsules in Solution and on Polymeric Surfaces. Adv. Mater. Interfaces 2017, 4, 1600273.

(44) Hsu, B. B.; Park, M. H.; Hagerman, S. R.; Hammond, P. T. Multimonth Controlled Small Molecule Release from Biodegradable Thin Films. Proc. Natl. Acad. Sci. U. S. A. 2014, 111, 12175-12180.

(45) Serpe, M. J.; Yarmey, K. A.; Nolan, C. M.; Lyon, L. A. Doxorubicin Uptake and Release from Microgel Thin Films. Biomacromolecules 2005, 6, 408-413.

(46) Chung, A. J.; Rubner, M. F. Methods of Loading and Releasing Low Molecular Weight Cationic Molecules in Weak Polyelectrolyte Multilayer Films. Langmuir 2002, 18, 1176-1183.

(47) Prokopovic, V. Z.; Duschl, C.; Volodkin, D. Hyaluronic Acid/ Poly-l-Lysine Multilayers as Reservoirs for Storage and Release of Small Charged Molecules. Macromol. Biosci. 2015, 15, 1357-1363.

(48) Zoghbi, M. E.; Cooper, R. S.; Altenberg, G. A. The Lipid Bilayer Modulates the Structure and Function of an ATP-binding Cassette Exporter. J. Biol. Chem. 2016, 291, 4453-4461.

(49) Pyo, M.; Reynolds, J. R. Electrochemically Stimulated Adenosine 5'-Triphosphate (ATP) Release Through Redox Switching of Conducting Polypyrrole Films and Bilayers. Chem. Mater. 1996, 8, $128-133$.

(50) Noguchi, T.; Shiraki, T.; Dawn, A.; Tsuchiya, Y.; Lien, L. T. N.; Yamamoto, T.; Shinkai, S. Nonlinear Fluorescence Response Driven by ATP-Induced Self-Assembly of Guanidinium-Tethered Tetraphenylethene. Chem. Commun. 2012, 48, 8090-8092.

(51) Aisawa, S.; Ohnuma, Y.; Hirose, K.; Takahashi, S.; Hirahara, H.; Narita, E. Intercalation of Nucleotides into Layered Double Hydroxides by Ion-Exchange Reaction. Appl. Clay Sci. 2005, 28, 137-145.

(52) Singh, P.; Solomon, V. C.; Buttry, D. A. Electrochemistry of ATP-Capped Silver Nanoparticles in Layer-by-Layer Multilayer Films. J. Nanopart. Res. 2014, 16, 2496.

(53) Yan, Q.; Zhao, Y. ATP-Triggered Biomimetic Deformations of Bioinspired Receptor-Containing Polymer Assemblies. Chem. Sci. 2015, 6, 4343-4349.

(54) Picart, C.; Mutterer, J.; Richert, L.; Luo, Y.; Prestwich, G. D.; Schaaf, P.; Voegel, J. C.; Lavalle, P. Molecular Basis for the Explanation of the Exponential Growth of Polyelectrolyte Multilayers. Proc. Natl. Acad. Sci. U. S. A. 2002, 99, 12531-12535.

(55) Vikulina, A. S.; Anissimov, Y. G.; Singh, P.; Prokopovic, V. Z.; Uhlig, K.; Jaeger, M. S.; von Klitzing, R.; Duschl, C.; Volodkin, D. Temperature Effect on the Build-Up of Exponentially Growing Polyelectrolyte Multilayers. An Exponential-to-Linear Transition Point. Phys. Chem. Chem. Phys. 2016, 18, 7866-7874.

(56) Volodkin, D.; von Klitzing, R. Competing Mechanisms in Polyelectrolyte Multilayer Formation and Swelling: PolycationPolyanion Pairing vs. Polyelectrolyte-Ion Pairing. Curr. Opin. Colloid Interface Sci. 2014, 19, 25-31.

(57) Madaboosi, N.; Uhlig, K.; Jaeger, M. S.; Moehwald, H.; Duschl, C.; Volodkin, D. V. Microfluidics as A Tool to Understand the BuildUp Mechanism of Exponential-Like Growing Films. Macromol. Rapid Commun. 2012, 33, 1775-1779.

(58) Schmidt, S.; Madaboosi, N.; Uhlig, K.; Koehler, D.; Skirtach, A.; Duschl, C.; Moehwald, H.; Volodkin, D. V. Control of Cell Adhesion by Mechanical Reinforcement of Soft Polyelectrolyte Films with Nanoparticles. Langmuir 2012, 28, 7249-7257.

(59) Prokopovic, V. Z.; Duschl, C.; Volodkin, D. V. Hyaluronic Acid/ Poly-L-Lysine Multilayers Coated with Gold Nanoparticles: Cellular Response and Permeability Study. Polym. Adv. Technol. 2014, 25, $1342-1348$.

(60) Sustr, D.; Duschl, C.; Volodkin, D. A FRAP-Based Evaluation of Protein Diffusion in Polyelectrolyte Multilayers. Eur. Polym. J. 2015, 68, 665-670.

(61) Seiffert, S.; Oppermann, W. Systematic Evaluation of FRAP Experiments Performed in a Confocal Laser Scanning Microscope. J. Microsc. 2005, 220, 20-30.

(62) Crouzier, T.; Picart, C. Ion Pairing and Hydration in Polyelectrolyte Multilayer Films Containing Polysaccharides. Biomacromolecules 2009, 10, 433-442.

(63) Bonizzoni, M.; Long, S. R.; Rainwater, C.; Anslyn, E. V. PAMAM Dendrimer-Induced Aggregation of 5(6)-Carboxyfluorescein. J. Org. Chem. 2012, 77, 1258-1266.

(64) Graber, M. L.; Dilillo, D. C.; Friedman, B. L.; Pastorizamunoz, E. Characteristics of Fluoroprobes for Measuring Intracellular $\mathrm{pH}$. Anal. Biochem. 1986, 156, 202-212.

(65) Jourdainne, L.; Lecuyer, S.; Arntz, Y.; Picart, C.; Schaaf, P.; Senger, B.; Voegel, J. C.; Lavalle, P.; Charitat, T. Dynamics of Poly(LLysine) in Hyaluronic Acid/Poly(L-Lysine)Multilayer Films Studied by Fluorescence Recovery After Pattern Photobleaching. Langmuir 2008, 24, 7842-7847.

(66) Limousin, G.; Gaudet, J. P.; Charlet, L.; Szenknect, S.; Barthes, V.; Krimissa, M. Sorption Isotherms: A Review on Physical Bases, Modeling and Measurement. Appl. Geochem. 2007, 22, 249-275.

(67) Hill, L.; Flack, M. The Influence of Oxygen Inhalations on Muscular Work. J. Physiol. 1910, 40, 347-372.

(68) Burke, S. E.; Barrett, C. J. pH-Responsive Properties of Multilayered Poly(L-Lysine)/Hyaluronic Acid Surfaces. Biomacromolecules 2003, 4, 1773-1783. 
(69) Shchukin, D. G.; Shutava, T.; Shchukina, E.; Sukhorukov, G. B.; Lvov, Y. M. Modified Polyelectrolyte Microcapsules as Smart Defense Systems. Chem. Mater. 2004, 16, 3446-3451. 